УДК 339.5:330.4(470)

https://doi.org/10.24866/1813-3274/2020-3/68-88

Н. В. Кузнецова ${ }^{1}$, Дальневосточный федеральный университет,

г. Владивосток, Россия

E-mail: kuznetsova.nv@dvfu.ru

E. В. Кочева ${ }^{2}$, Дальневосточный федеральный университет, г. Владивосток, Россия E-mail: kocheva.ev@dvfu.ru

Н. А. Матев ${ }^{3}$, Дальневосточный федеральный университет, г. Владивосток, Россия E-mail: matev.na@dvfu.ru

\title{
РИСК ПОТЕРИ ПОТЕНЦИАЛА ВЗАИМНЫХ ТОРГОВЫХ ПОТОКОВ СУБЬЕКТОВ РОССИЙСКОЙ ФЕДЕРАЦИИ: ЭКОНОМЕТРИЧЕСКОЕ МОДЕЛИРОВАНИЕ
}

Аннотация. Особую актуальность в современных условиях приобретают вопросы оценки влияния внутренних социально-экономических факторов на минимизацию риска потери потенциала взаимных торговых потоков субъектов России со странами-партнёрами. Данный риск был выделен и количественно оценен в рамках большого вызова, стоящего перед Российской Федерацией по преодолению консервативности внешнеэкономической деятельности с целью догнать странылидеры по продвижению продукции на мировые рынки. Для определения ключевых факторов, оказывающих влияние на риск потери потенциала торговых потоков,

\footnotetext{
${ }^{1}$ Наталия Викторовна Кузнецова, доктор экономических наук, профессор, профессор кафедры мировой экономики, Школа экономики и менеджмента, Дальневосточный федеральный университет, г. Владивосток, Россия.

${ }^{2}$ Екатерина Викторовна Кочева, кандидат экономических наук, доцент кафедры бизнес-информатики и экономико-математических методов, Школа экономики и менеджмента, Дальневосточный федеральный университет, г. Владивосток, Россия.

${ }^{3}$ Николай Анатольевич Матев, кандидат экономических наук, доцент кафедры бизнес-информатики и экономико-математических методов, Школа экономики и менеджмента, Дальневосточный федеральный университет, г. Владивосток, Россия.

Для цитирования: Кузнецова Н. В., Кочева Е. В., Матев Н. А. Риск потери потенциала взаимных торговых потоков субъектов Российской Федерации: эконометрическое моделирование // Азиатско-Тихоокеанский регион: экономика, политика, право. 2020. № 3. С. 68-88. https://doi.org/10.24866/1813-3274/2020-3/68-88.

* Результаты были получены при выполнении научного проекта Российского фонда фундаментальных исследований (РФФИ) № 18-014-00001 «Модель мультивекторной социально-экономической политики взаимодействия российского Дальнего Востока и стран АТР - пути снижения неожиданных эффектов от наступления «больших вызовов».
}

(C) Кузнецова Н. В., Кочева Е. В., Матев Н. А., 2020 
был сформирован априорный набор статистических показателей. С помощью корреляционного анализа была рассчитана матрица парных коэффициентов корреляции и был сформирован апостериорный набор предикторов, оказывающих набольшее влияние на зависимую переменную. В статье представлены три модели: сквозной регрессии, с фиксированными эффектами, со случайными эффектами. Представленное сравнение трёх построенных моделей показало, что наиболее предпочтительной для описания изменчивости риска потери потенциала взаимных торговых потоков от выделенных факторов является модель с фиксированными эффектами. Авторами были построены детализированные модели с фиксированными эффектами с учётом влияния индивидуальных особенностей субъектов России, временных эффектов, а также модель, учитывающая их одновременное влияние. Доказано, что безусловно индивидуальные эффекты субъектов РФ оказывают значимое влияние на изменение риска потери потенциала взаимных торговых потоков, в особенности это является актуальным для Дальневосточного федерального округа (ДФО) ввиду неразвитости инфраструктуры, отдалённости от центральной части России, высокими миграционными оттоками постоянного населения в сочетании с его естественной убылью, недостаточным уровнем жизни населения, а также низкой покупательной способностью жителей региона.

Ключевые слова: риск, модель, торговые потоки, сквозная модель регрессии, модель с фиксированными эффектами, модель со случайными эффектами, тест Хаусмана, тест Бройша-Пагана, дамми-переменные, инвестиции, ДФО, АТР, уровень бедности, реальные денежные доходы, уровень безработицы. 
Natalia V. Kuznetsova ${ }^{1}$, Far Eastern Federal University, Vladivostok, Russia E-mail: kuznetsova.nv@dvfu.ru

Ekaterina V. Kocheva ${ }^{2}$, Far Eastern Federal University, Vladivostok, Russia E-mail: kocheva.ev@dvfu.ru

Nikolay A. Matev ${ }^{3}$, Far Eastern Federal University, Vladivostok, Russia

E-mail: matev.na@dvfu.ru

\section{RISK OF POTENTIAL LOSS OF MUTUAL TRADE FLOWS OF REGIONS OF THE RUSSIAN FEDERATION: ECONOMETRIC MODELING*}

Abstract. The special relevant features in modern conditions have the issues of assessing the influence of internal socio-economic factors on minimizing the risk of losing the potential of mutual trade flows of Russian regions with partner countries. This risk was identified and quantified within the framework of the grand challenge which facing the Russian Federation in overcoming the conservatism of foreign economic activity in order to catch up with the leading countries in promoting products to world markets. In order to determine the key factors affecting the risk of losing the potential of trade flows, a priori set of statistical indicators was formed. Using correlation analysis, a matrix of paired correlation coefficients was calculated and a posteriori set of predictors that had the greatest impact on the dependent variable was formed. The article presents three models: sequentially regression, fixed effects, and random effects. The presented comparison of three constructed models showed that the most preferable for describing the variability of the risk of losing the potential of mutual trade flows from the selected factors is the model with fixed effects. The authors constructed detailed models with fixed effects, taking into account the influence of individual characteristics of the regions of Russia, time effects, as well as a model that take into account its simultane-

\footnotetext{
${ }^{1}$ Natalia V. Kuznetsova, Doctor of Economics, Professor, International Economics Department, School of Economics and Management, Far Eastern Federal University, Vladivostok, Russia.

${ }^{2}$ Ekaterina V. Kocheva, Candidate of Economic Sciences, Associate Professor, Department of Business Informatics, Economic and Mathematical Methods, School of Economics and Management, Far Eastern Federal University, Vladivostok, Russia.

${ }^{3}$ Nikolay A. Matev, Candidate of Economic Sciences, Associate Professor, Department of Business Informatics, Economic and Mathematical Methods, School of Economics and Management, Far Eastern Federal University, Vladivostok, Russia.

For citing: Kuznetsova N. V., Kocheva E. V., Matev N. A. Risk of potential loss of mutual trade flows of regions of the Russian Federation: econometric modeling // Pacific RIM: Economics, Politics, Law. 2020. No. 3. P. 68-88. https://doi.org/10.24866/1813-3274/2020-3/68-88.

* The results were obtained during the implementation of the scientific project of the Russian Foundation for Basic Research (RFBR) No. 18-014-00001 "Model of a multi-vector socio-economic policy of interaction between the Russian Far East and the APR countries - ways to reduce unexpected effects from the onset of "large calls".
} 
ous influence. It was proved that the individual effects of the regions of the Russian Federation have a significant impact on the changing in the risk of losing the potential of mutual trade flows, it is especially relevant for the Far Eastern Federal District due to the underdeveloped infrastructure, remoteness from the central part of Russia, high migration outflows of the resident population, combined with its natural declining process, insufficient standard of living of the population, as well as the low purchasing power of the inhabitants of the region.

Keywords: risk, model, trade flows, sequentially regression model, fixed effects model, random effects model, the Hausman test, the Breusch-Pagan test, dummy variables, investment, FEFD, APR, poverty rate, real incomes, unemployment rate.

\section{Введение}

В современных условиях ни одно государство не способно развиваться без внешнеторговой деятельности, значительно возрастает роль внешней торговли в развитии национальной экономики страны. Внешняя торговля считается одним из приоритетных направлений, способствующих становлению и стабилизации экономики страны, а также росту благосостояния нации. Рассмотрение проблематики формирования и развития внешнеторговых отношений имеет большую историю.

Ф. Родригес и Д. Родрик утверждают, что международная торговая политика тесно связана со многими другими аспектами экономической политики и это усложняет определение степени влияния международной торговли на экономический рост [1]. Представители неоклассического направления Э. Хекшер [2] и Б. Олин [3] в факторной теории внешней торговли показали, что внешняя торговля приводит к увеличению доходов владельцев относительно избыточных факторов производства и экспорта продукта и, как следствие, стимулирует экономический рост.

Многие исследования воздействия открытости торговли и прямых иностранных инвестиций (ПИИ) на экономический рост в рамках модели ELGH не дают однозначных эмпирических результатов. В частности, Balasubramanyam и др. [4] на основе регрессионного анализа обосновали взаимосвязь открытости внешней торговли с эффективностью воздействия ПИИ на экономический рост в развивающихся странах. Р. Вачарг также показал, что международная торговля влияет на экономический рост посредством прямых иностранных инвестиций [5]. Caudros и др. [6] использовал модель авторегрессии для обоснования наличия причинноследственной связи экономического роста с притоком прямых иностранных инвестиций и внешней торговлей, в исследовании подтвердилась гипотеза FLGH.

Результаты эконометрического анализа Baliamoune-Lutz [7] показали наличие двусторонней взаимосвязи между экспортом и прямыми иностранными инвестициями, позитивно влияющими на динамику экономического роста.

Однако Hisarciklilar и др. [8], изучавшие взаимосвязь между экономическим ростом, ПИИ и торговлей с применением коинтеграции Энгла и тестов Грейнд- 
жера, так же как и Darrat и др. [9], не обнаружили такой причинно-следственной связи в большинстве средиземноморских стран.

Alaya [10], обнаружил, что экономический рост большей частью определяется динамикой экспорта и внутренних инвестиций и в меньшей степени - параметрами человеческого капитала. Однако Дж. Франкель и Д. Ромер ещё в 1999 г. специфицировали влияние торговли на экономический рост посредством фактора накопления человеческого капитала [11].

Ещё один метод статистического анализа - анализ причинности, который использует либо одно уравнение регрессии Гранжера, либо множественную векторную авторегрессию (VAR). $V A R$ модели постоянно используются для оценки воздействия международной торговли на экономический рост. Ряд исследований использовали уравнения регрессии, в которых все переменные приведены в уровнях, а не по темпам роста. В этой связи Дж. Франкель и Д. Ромер заявляют, что большие различия между уровнями переменных позволяют более точно оценивать регрессию [11].

В нашем исследовании были разработаны эффективные формы взаимодействия Дальнего Востока России и стран Азиатско-Тихоокеанского региона и выделены четыре основные вида экономической деятельности по следующей номенклатуре товарных групп: 03 - Рыба и ракообразные, моллюски и прочие водные беспозвоночные; 27 - Топливо минеральное, нефть и продукты их перегонки; битуминозные вещества; воски минеральные; 44 - Древесина и изделия из неё; древесный уголь; 84 - Реакторы ядерные, котлы, оборудование и механические устройства; их части. Как было определено на предыдущем этапе исследования, основными торговыми партнёрами ДФО выступают следующие страны АТР: Китайская Народная Республика, Тайвань, Таиланд, США, Республика Корея, Япония, Канада, Сингапур, Индонезия, Филиппины, Малайзия и Вьетнам.

Ранее в исследовании «Методика оценки социально-экономических рисков для ответа России на большие вызовы» нами были определены и оценены уровни социально-экономических рисков для ответа России на большие вызовы. В частности, для оценки был сформирован набор показателей и разработана методика, позволяющая количественно оценить потенциальные риски [12]. Были проанализированы внешнеэкономические торговые отношения Дальнего Востока России и стран-партнёров Азиатско-Тихоокеанского региона в рамках разработанных моделей эффективных международных форм взаимодействий, а также выделены основополагающие факторы, оказывающие влияние на стоимостные объёмы экспортноимпортных операций [13].

Особую актуальность, в продолжение исследования эффективных международных форм взаимодействия, приобретают вопросы оценки влияния внутренних социально-экономических факторов на изменение (а конкретнее - на минимиза- 
цию) риска потери потенциала взаимных торговых потоков субъектов России со странами-партнёрами. Данный риск был выделен и количественно оценен в рамках большого вызова, стоящего перед Российской Федераций по преодолению консервативности внешнеэкономической деятельности с целью догнать страны-лидеры по продвижению продукции на мировые рынки.

\section{Методика исследования}

Для определения ключевых факторов, оказывающих влияние на риск потери потенциала торговых потоков, был сформирован априорный набор статистических показателей из 47 наименований (см. рис. 1).

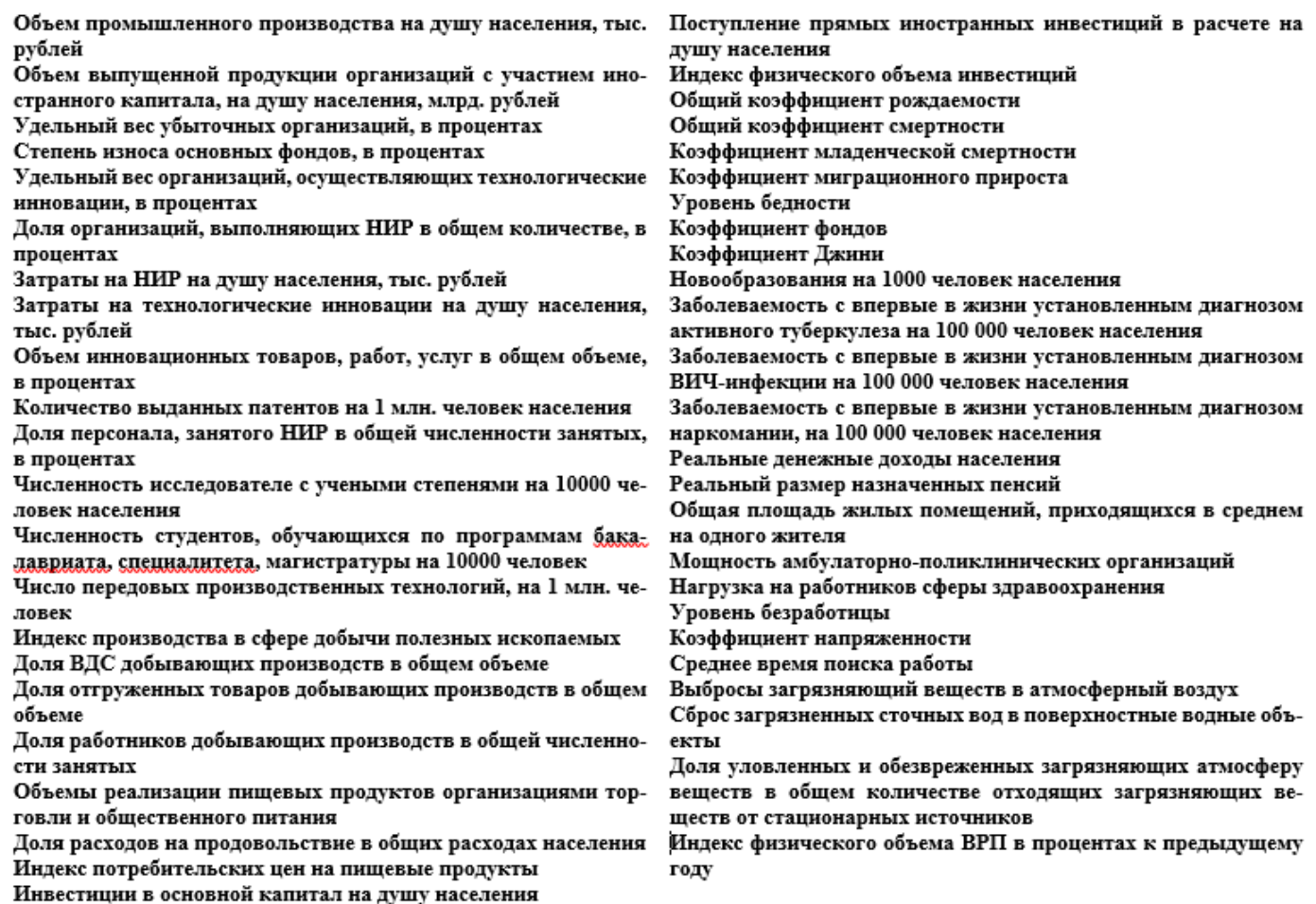

Инвестицин в основной капитал на душу населения

\section{Puc. 1. Набор статистических показателей.}

Источник: составлено авторами

Далее из априорного набора показателей с помощью корреляционного анализа была рассчитана матрица парных коэффициентов корреляции, характеризующая степень зависимости между исследуемыми переменными. Затем на основе анализа абсолютных значений коэффициентов корреляции факторов и зависимой переменной, в качестве которой выступил риск потери потенциала взаимных торговых потоков, был сформирован апостериорный набор предикторов, оказывающих набольшее влияние на зависимую переменную. Итоговый набор факторов представлен в табл. 1. 
Таблииа 1

Апостериорный набор предикторов для моделирования изменчивости риска потери потенциала взаимных торговых потоков

\begin{tabular}{|c|c|c|c|}
\hline Обозначение & $\begin{array}{c}\text { Типы } \\
\text { переменной }\end{array}$ & Наименование показателя & $\begin{array}{c}\text { Единицы } \\
\text { измерения }\end{array}$ \\
\hline risk & зависимая & $\begin{array}{l}\text { риск потери потенциала взаимных } \\
\text { торговых потоков в субъекте }\end{array}$ & - \\
\hline im_prom_pr & \multirow[t]{12}{*}{ факторные } & $\begin{array}{l}\text { объём промышленного производ- } \\
\text { ства на душу населения }\end{array}$ & тыс. руб. \\
\hline iznos_of & & степень износа основных фондов & $\%$ \\
\hline zatrati_nir & & затраты на НИР на душу населения & руб. \\
\hline index_potreb_tcen & & индекс потребительских цен & $\%$ \\
\hline invest_v_ok & & $\begin{array}{l}\text { инвестиции в основной капитал на } \\
\text { душу населения }\end{array}$ & руб. \\
\hline pryam_inosrt_inv & & $\begin{array}{l}\text { прямые иностранные инвестиции } \\
\text { на душу населения }\end{array}$ & $\begin{array}{l}\text { тыс. долл. } \\
\text { США }\end{array}$ \\
\hline migr_prirost & & $\begin{array}{l}\text { миграционный прирост } \\
\text { на } 10000 \text { чел. населения }\end{array}$ & чел. \\
\hline urov_bednosti & & уровень бедности & $\%$ \\
\hline real_den_doh_nas & & $\begin{array}{l}\text { реальные денежные доходы } \\
\text { населения }\end{array}$ & $\begin{array}{l}\text { \% к предыду- } \\
\text { щему году }\end{array}$ \\
\hline urov_bezrab & & уровень безработицы & $\%$ \\
\hline index_fix_ob_vrp & & индекс физического объёма ВРП & $\begin{array}{l}\text { \% к предыду- } \\
\text { щему году }\end{array}$ \\
\hline dobicha & & $\begin{array}{l}\text { дамми-переменная, принимающая } \\
\text { значение } 1 \text {, если основу экономики } \\
\text { региона составляют добывающие } \\
\text { производства, } 0 \text { - в остальных } \\
\text { случаях }\end{array}$ & - \\
\hline
\end{tabular}

Источник: составлено авторами

Структура исходной выборки представляет собой панельные данные за 8 лет в период 2010-2017 гг. по 80 субъектам Российской Федераций. Информационной базой для подготовки исходной выборки послужили данные Федеральной службы государственной статистики России. Таким образом мы получили сбалансированную панель, состоящую из 640 наблюдений.

Это позволило качественно анализировать распределение тех или иных эффектов как во времени, так и с учётом индивидуальных ненаблюдаемых характеристик субъектов России, которые не меняются или незначительно меняются во вре- 
мени и принимают различные значения для каждого конкретного региона (например, климатические условия, удалённость региона от центра страны, развитость инфраструктуры и т.п.). Специфические особенности каждого субъекта при этом учитывались через индивидуальные эффекты, устойчивые на протяжении всего рассматриваемого периода времени.

Реализация оценок эконометрических моделей проводилась с помощью языка программирования $R$ в среде Rstudio. Перед проведением эконометрического анализа были рассчитаны описательные статистики по каждому из представленных в апостериорном наборе показателей в разрезе исследуемых периодов (2010-2017 гг.)

\section{Результаты исследования}

В начале эконометрического исследования рассмотрим модель сквозной регрессии, в которой предполагается, что индивидуальные особенности субъектов России и временные эффекты не оказывают влияние на риск потери потенциала взаимных торговых потоков, таким образом модель сквозной регрессии предписывает одинаковое поведение всем объектам выборки во все периоды времени. Также отметим, что в качестве спецификации модели была выбрана линейная спецификация в связи с тем, что по ней лучше всего интерпретировать влияние исходного набора предикторов. На рис. 2 представлена сквозная модель регрессии.

Согласно данным рис. 2, можно сказать, что все выделенные предикторы оказывают значимое влияние как минимум при 10\% уровне значимости. В целом построенная модель объясняет изменчивость риска потери потенциала взаимных торговых потоков на 64,33\% и является статистически значимой при любом разумном уровне значимости согласно $F$-критерию Фишера ( $p$-value $<2.22 \mathrm{e}-16)$.

Отметим, что положительное влияние на риск потери потенциала взаимных торговых потоков оказывают следующие факторы: объёмы промышленного производства в расчёте на душу населения, затраты на НИР, прямые иностранные инвестиции, миграционный прирост, а также факторы, отвечающие за производственный потенциал и добычу полезных ископаемых: при их росте значение показателя риска будет увеличиваться, a, следовательно, согласно нашей методике построения данного показателя, риск будет снижаться. Знаки при данных коэффициентах не противоречат действительности.

Отрицательное значимое влияние на изменчивость результативного показателя (тем самым провоцирующее увеличение риска потери потенциала взаимных торговых потоков субъектов России и стран) оказывают предикторы: уровень износа основных фондов, доля инновационных товаров в общем объёме, индекс потребительских цен, объёмы инвестиций в основной капитал в расчёте на душу населения, уровни бедности и безработицы, а также темпы ростов реальных денежных доходов населения. 
Pooling Model

Cal1:

plm(formula $=$ risk $\sim$ im_prom_pr + iznos_of + share_intov + zatrati_nir + index_potreb_tcen + invest_v_ok + pryam_inosrt_in + migr_prirost + urov_bednosti + real_den_doh_nas + urov_bezrab + index_fix_ob_vrp + dobicha, data $=$ data_pane 1 , mode $1=$ "pooling")

Balanced Pane1: $n=80, T=8, N=640$

Residuals:

Min. 1st Qu. Median 3rd Qu. Max.

$\begin{array}{lllll}-0.2963061 & -0.0449416 & 0.0041542 & 0.0461709 & 0.3540675\end{array}$

Coefficients:

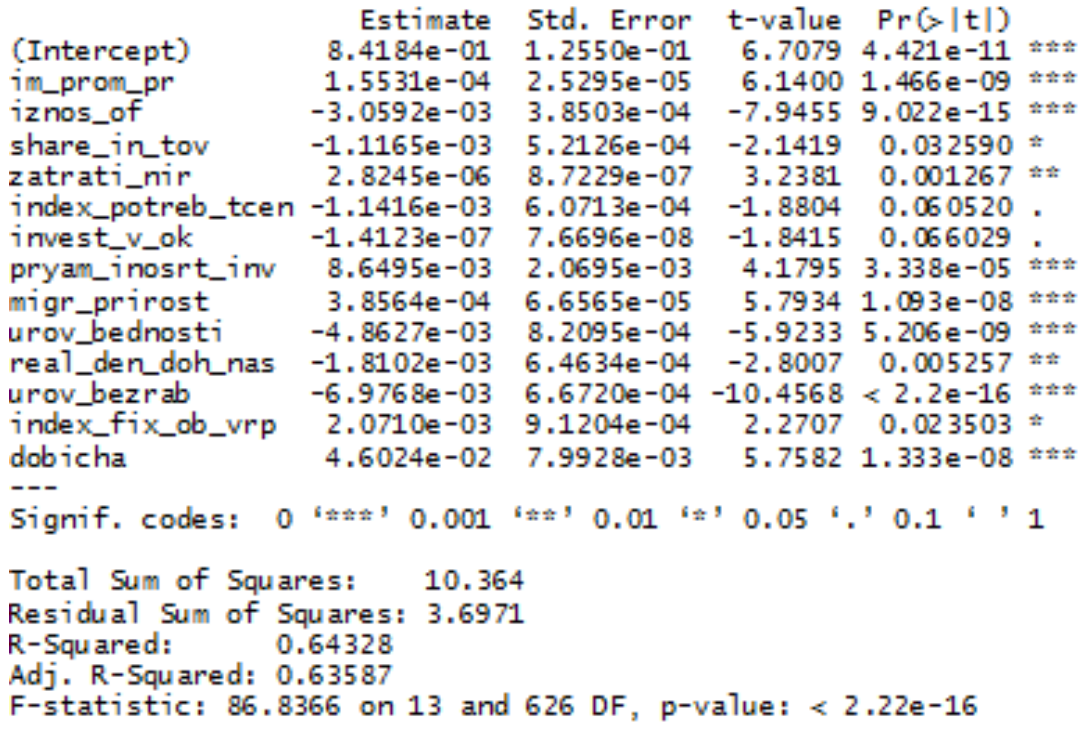

Puc. 2. Модель сквозной регрессии зависимости риска потери потенциала взаимных торговых потоков от выделенных факторов.

Источник: расчёты авторов

Исходя из результатов оценивания данной эконометрической модели возникает противоречивая ситуация, когда увеличение потока инвестиций в основной капитал и доли инновационной продукции в общем объёме приводят к увеличению риска потери потенциала взаимных торговых потоков. Чтобы проверить, не вызван ли этот неожиданный результат смещением оценки данных коэффициентов, оценим модель с фиксированными эффектами (рис. 3).

Мы видим, что в числе незначимых оказались следующие факторы: доля инновационных товаров в общем объёме, индекс потребительских цен и индекс физического объёма валового регионального продукта. В целом оставшиеся факторы оказывают значимое влияние на зависимую переменную, а их знаки аналогичны знакам из предыдущей модели. Отметим, что модель учитывает общие для всех субъектов России струк- 
Кузнецова Н. В., Кочева Е. В., Матев Н. А. Риск потери потенциала взаимных торговых потоков субъектов Российской Федерации: эконометрическое моделирование

турные изменения, которые происходят с течением времени. Кроме этого видно, что модель с фиксированными эффектами имеет лучшее качество как согласно значению коэффициента детерминации, так и значению исправленного коэффициента детерминации. В целом с помощью данной модели удалось объяснить 71,97\% изменчивости зависимой переменной, а в силу того, что значение $p$-value критерия Фишера достаточно мало, следует принять гипотезу о значимости всей модели регрессии в целом.

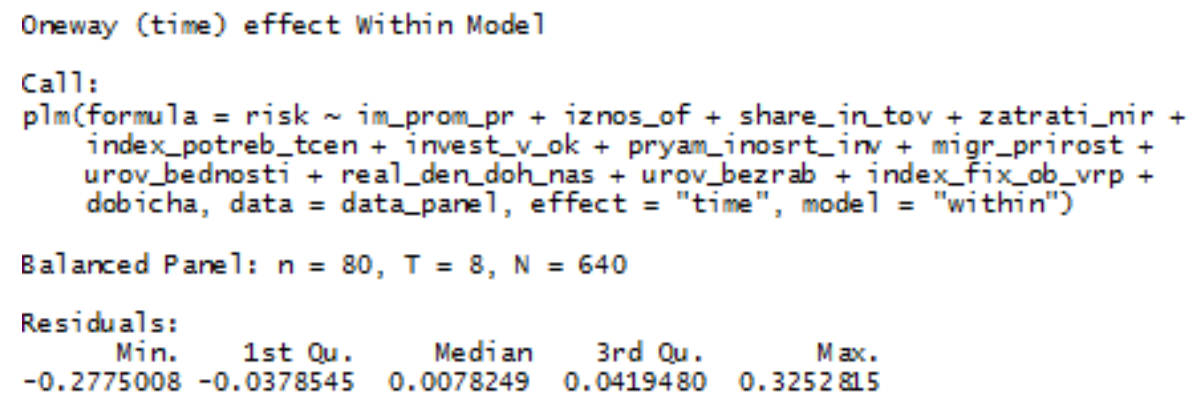

Coefficients:

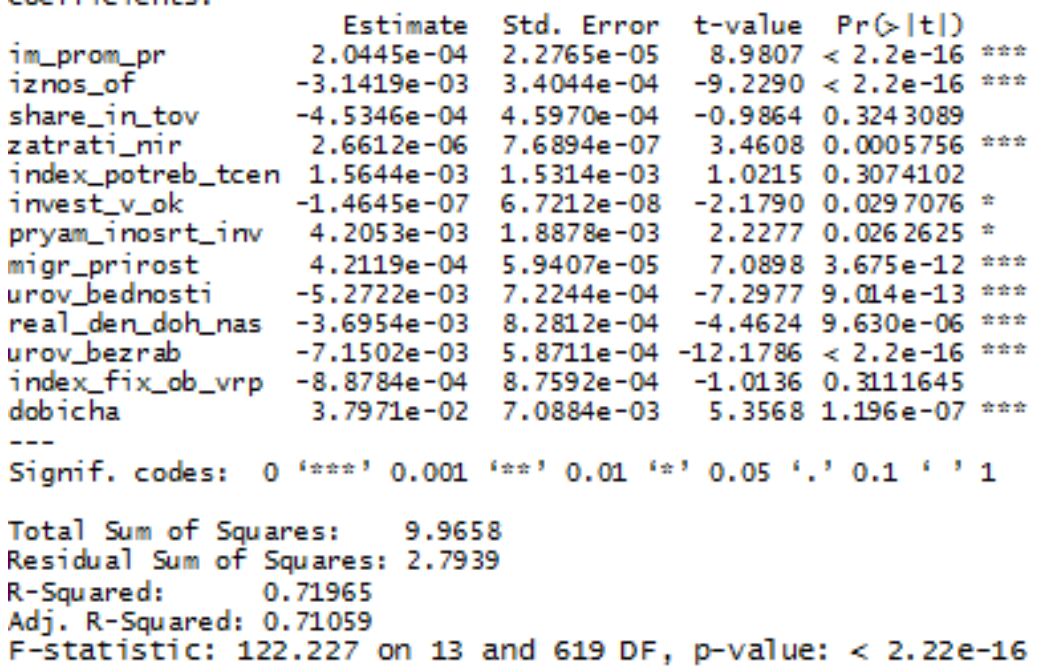

Puc. 3. Модель регрессии с фиксированными эффектами зависимости риска потери потенциала взаимных торговых потоков от выделенных факторов.

Источник: расчёты авторов

Оценим третью модель - модель со случайными эффектами (см. рис. 4). Её смысл заключается в том, что она отражает влияние ненаблюдаемых или пропущенных в модели переменных, которые характеризуют индивидуальные особенности субъектов Российской Федерации. Стоит заметить, что данные индивидуальные различия носят теперь случайный характер и в среднем сглаживаются. 


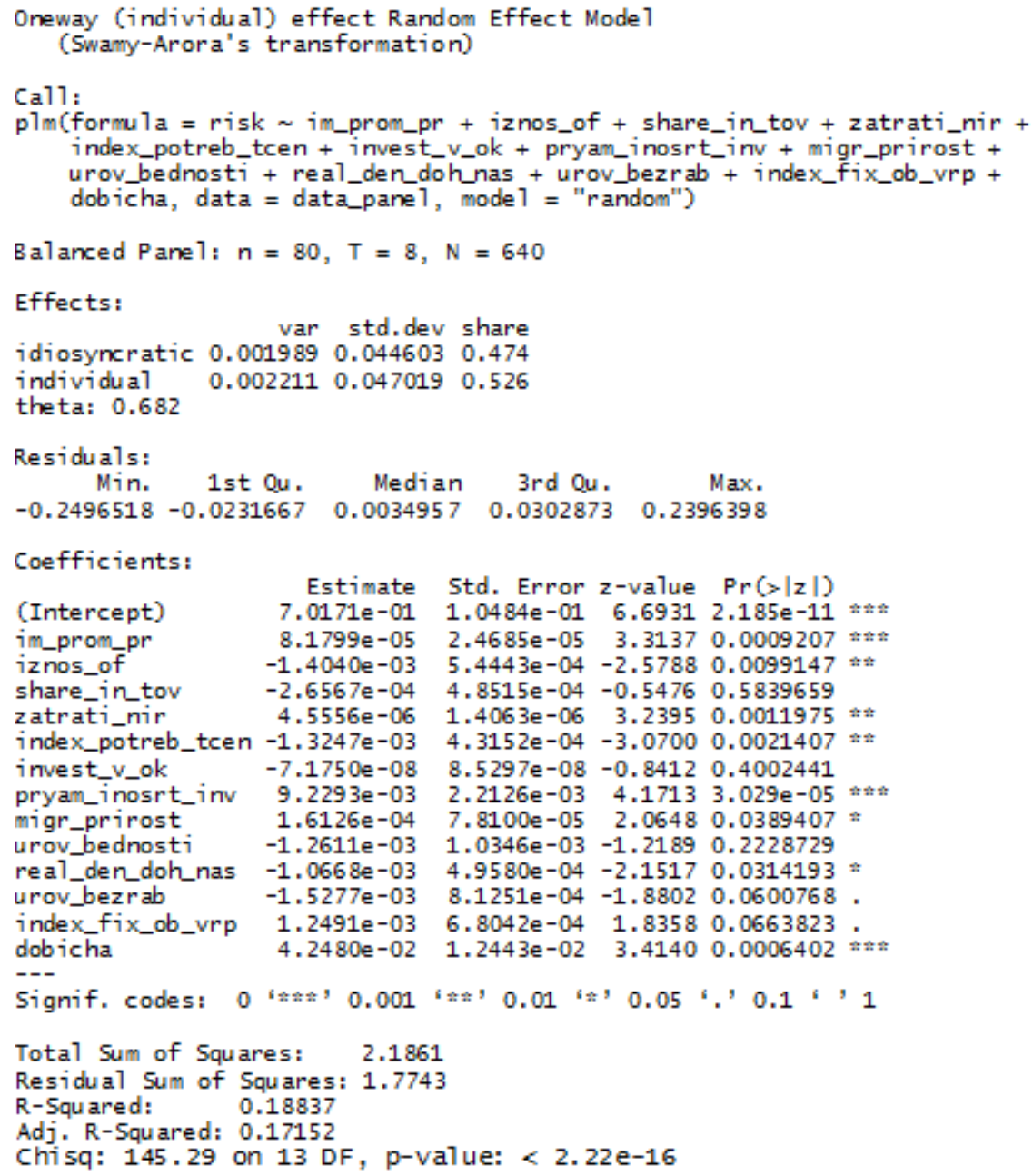

Puc. 4. Модель регрессии со случайными эффектами зависимости риска потери потенциала взаимных торговых потоков от выделенных факторов.

Источник: расчёты авторов

В числе незначимых факторов выделились следующие: доля инновационных товаров в общем объёме, инвестиции в основной капитал и уровень бедности. Отметим, что в модели со случайными эффектами невозможно интерпретировать значение коэффициента детерминации и он не является адекватной мерой качества построенного уравнения. Значимость построенной эконометрической модели определяет статистика Вальда Chisq=145,29.

Результаты оценок уравнения со случайными эффектами отличаются от результатов, полученных в предыдущей модели с фиксированными эффектами. Так снизилась статистическая значимость показателей износа основных фондов, затрат на НИР, 
миграционного прироста, темпов прироста денежных доходов населения, а также уровня безработицы, т.е. данные факторы в этой модели не оказывают серьёзного влияния, по сравнению с предыдущей моделью, на риск потери потенциала взаимных торговых потоков. При этом стоит отметить, что значимость иностранных инвестиций выросла, а также значимым стал показатель индекса потребительских цен.

На следующем этапе проведем сравнение трёх построенных моделей и выберем одну, которая наилучшим образам описывает изменчивость риска потери потенциала взаимных торговых потоков в субъектах Российской Федерации.

Проведём $F$-тест для определения предпочтительного уравнения регрессии между уравнением сквозной регрессии и моделью с фиксированными эффектами (рис. 5).

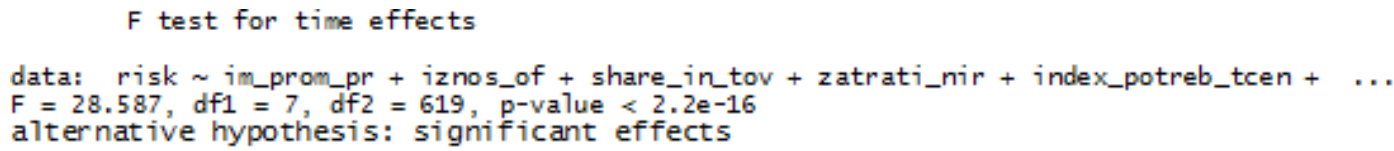

Puc. 5. Результаты $F$-теста сравнения моделей сквозной регрессии и регрессии с фиксированными эффектами.

Источник: расчёты авторов

Как видно из рисунка 5, p-value теста Фишера не превышает уровня значимости в $10 \%$, следовательно нулевую гипотезу о верной сквозной модели необходимо отвергнуть в пользу модели с фиксированными эффектами.

Выбор между уравнением сквозной регрессии и моделью со случайными эффектами осуществляется с помощью теста Бройша-Пагана, который по сути является одним из тестов множителей Лагранжа. Нулевая гипотеза этого теста заключается в том, что дисперсия случайных эффектов равна нулю, таким образом можно сказать, что все объекты в выборке являются однородными. Результаты сравнения моделей представлены на рис. 6.

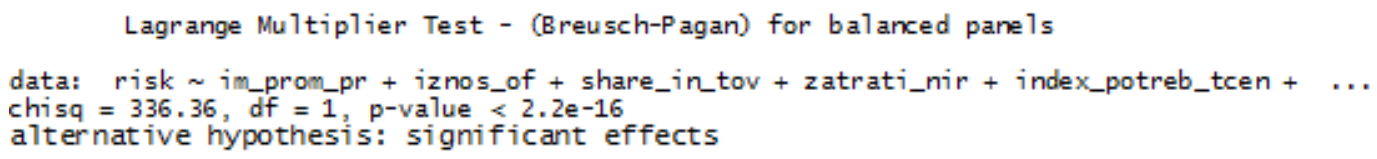

Puc. 6. Результаты теста Бройша-Пагана сравнения моделей сквозной регрессии и регрессии со случайными эффектами.

Источник: расчёты авторов

Значение наблюдаемой статистики достаточно велико, а значение p-value <2,2е-16. Таким образом, нулевая гипотеза отвергается в пользу модели со случайными эффектами, а модель сквозной регрессии не является предпочтительной по отношению к моделям с фиксированными и случайными эффектами. 
Для выбора лучшей из оставшихся двух моделей воспользуемся тестом Хаусмана. Результаты тестирования представлены на рис. 7.

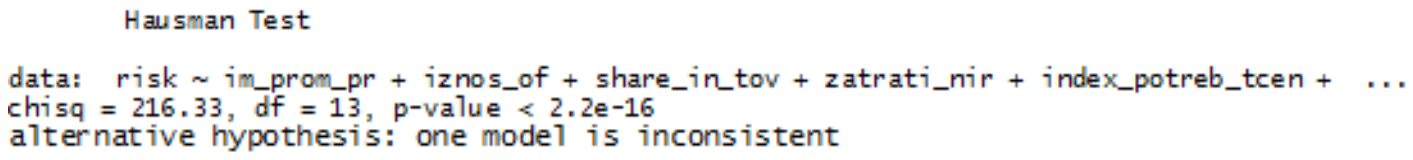

Рис. 7. Результаты теста Хаусмана сравнения моделей с фиксированными и со случайными эффектами.

Источник: расчёты авторов

Итоги теста Хаусмана свидетельствуют о том, что модель с фиксированными эффектами предпочтительней модели со случайными эффектами: значение рассчитанной статистики позволяет отвергнуть нулевую гипотезу о том, что ОМНК оценки модели со случайными эффектами являются состоятельными.

Таким образом, из представленных трёх моделей наиболее предпочтительной для описания изменчивости риска потери потенциала взаимных торговых потоков от выделенных факторов является модель с фиксированными эффектами. В числе незначимых оказались следующие факторы: доля инновационных товаров в общем объеме, индекс потребительских иен и индекс физического объёма валового регионального продукта.

Рассмотрим различные формы записи модели с фиксированными эффектами зависимости риска потери потенциала взаимных торговых потоков от выделенных факторов. Построим модели с фиксированными эффектами, добавив в качестве факторов дамми-переменные, отвечающие за период наблюдения, а также дамми-переменные, характеризующие принадлежность объекта выборки к субъекту Российской Федерации.

Во избежание полной мультиколлинеарности в качестве базовых категорий были выделены 2010 год - при введении дамми-переменных, отвечающих за временную составляющую, и г. Москва - при введении дамми-переменных, характеризующих субъект России.

Рассмотрим развёрнутую модель с фиксированными эффектами, характеризующую зависимость риска потери потенциала взаимных торговых потоков от выделенных факторов, с учётом временных периодов. Техника построения данной модели базируется на построении модели сквозной регрессии с добавлением фиктивных переменных, характеризующих год, коэффициенты при основных предикторах являются абсолютно равными коэффициентам регрессии within, рассмотренной выше. В данном случае особую значимость приобретают оценки коэффициентов при временных дамми-переменных. Модель с фиксированными эффектами с учётом временных периодов представлена на рис. 8. 
Кузнецова Н. В., Кочева Е. В., Матев Н. А. Риск потери потенциала взаимных торговых потоков субъектов Российской Федерации: эконометрическое моделирование

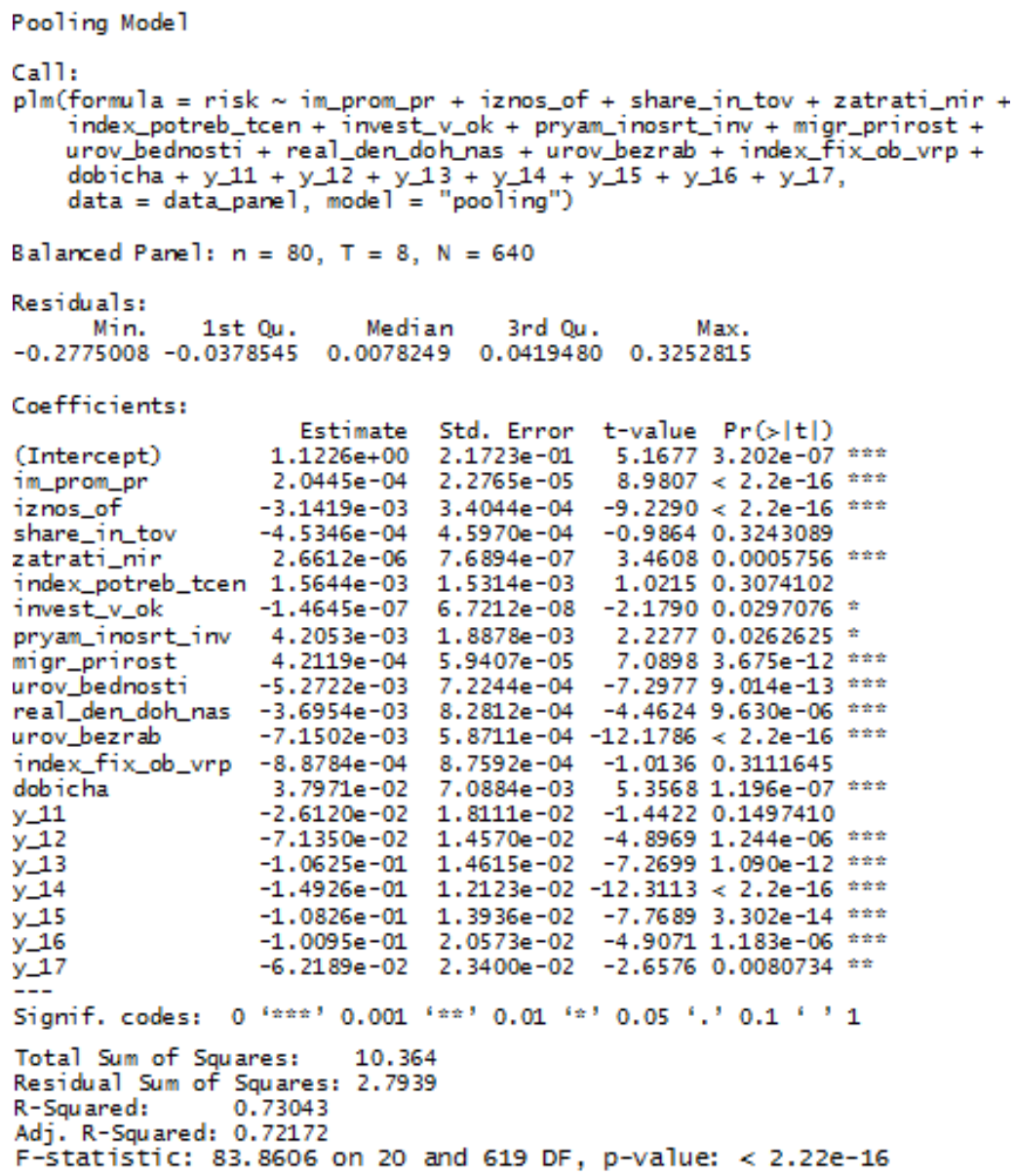

Рис. 8. Модель регрессии с фиксированными эффектами зависимости риска потери потенциала взаимных торговых потоков от выделенных факторов с учётом времени.

Источник: расчёты авторов

Как видно из рис. 8, оцененные регрессионные коэффициенты при даммипеременных, характеризующих период исследования, являются статистически значимыми за исключением 2011 г. Все полученные оценки имеют отрицательные значения, что свидетельствует о том, что с течением времени по отношению к базовой категории (2010 году) значение риска потери потенциала взаимных торговых потоков болыше, чем на начальный период исследования. Например, видно, что максимально отрицательное значение коэффициента среди всех дамми-переменных приходится на 2014 г.: по итогам расчётов значение составило минус 0,14926. Следовательно, этот период по сравнению с 2010 г. был наиболее кризисным в части риска потери потенциала взаимных торговых потоков. Это логично объясняется введением экономических санкций, что имело доста- 
точно серьёзные последствия в части притока иностранных инвестиций, а также для многих отраслей промышленности России, в том числе и топливно-энергетического комплекса. Всё это оказало влияние на внешнеторговые потоки страны и её субъектов в части их уменышения и, как следствие, увеличение риска потери их потенциала.

Отметим, что с течением времени данные негативные последствия ослабили свое влияние (значение коэффициентов в следующие периоды постепенно снижается). Данный факт обусловлен переориентацией российских внешнеторговых отношений в сторону стран АТР.

Аналогичным образом построим модель с фиксированными эффектами с учётом особенностей каждого субъекта Российской Федерации, поскольку индивидуальные особенности каждого субъекта играют важную роль в определении риска потери потенциала взаимных торговых потоков.

Регрессионная модель с учётом каждого субъекта представлена на рис. 9. Отметим, что часть дамми-переменных была скрыта ввиду громоздкости модели (79 дамми-переменных без учёта основных предикторов). Нас интересуют торговые потоки Дальневосточного федерального округа.

В числе значимых факторов, оказывающих влияние на риск потери потенциала взаимных торговых потоков, находятся индекс промышленного производства, уровень бедности населения в субъектах, темпы роста реальных денежных доходов населения, уровень безработицы. Отметим, что знаки при оценках коэффициентов не противоречат действительности, а выделенные предикторы характеризуют весьма типичные проблемы субъектов Дальнего Востока России. Особая роль при построении данной модели отводилась дамми-переменным, характеризующим индивидуальные особенности, присущие каждому субъекту Российской Федерации. Обозначения субъектов в модели представлены в табл. 2.

Таблицча 2

Обозначения субъектов ДФО в модели с фиксированными эффектами

\begin{tabular}{|l|l|l|l|}
\hline Обозначение & \multicolumn{1}{|c|}{$\begin{array}{c}\text { Субъект } \\
\text { Дальнего Востока России }\end{array}$} & Обозначение & \multicolumn{1}{c|}{$\begin{array}{c}\text { Субъект } \\
\text { Дальнего Востока Росси }\end{array}$} \\
\hline$s \_61$ & Республика Бурятия & $s_{-} 76$ & Амурская область \\
\hline$s \_65$ & Забайкальский край & $s_{-} 77$ & Магаданская область \\
\hline$s \_72$ & Республика Саха (Якутия) & $s_{-} 78$ & Сахалинская область \\
\hline$s \_73$ & Камчатский край & $s_{-79}$ & Еврейская автономная область \\
\hline$s \_74$ & Приморский край & $s_{-} 80$ & Чукотский автономный округ \\
\hline$s \_75$ & Хабаровский край & & \\
\hline
\end{tabular}

Источник: составлено авторами

Рассмотрим оценки коэффициентов при дамми-переменных, характеризующих субъекты ДФО. В соответствии с нашими расчётами, в число субъектов с наиболее 
Кузнецова Н. В., Кочева Е. В., Матев Н. А. Риск потери потенциала взаимных торговых потоков субъектов Российской Федерации: эконометрическое моделирование

высокими рисками потери потенциала взаимных торговых потоков по сравнению с базовым субъектом (г. Москва) вошли Еврейская автономная область, Забайкальский край, Республика Бурятия и Амурская областью. Меньшими рисками в ДФО обладают Сахалинская область, Приморский край и Чукотский автономный округ.

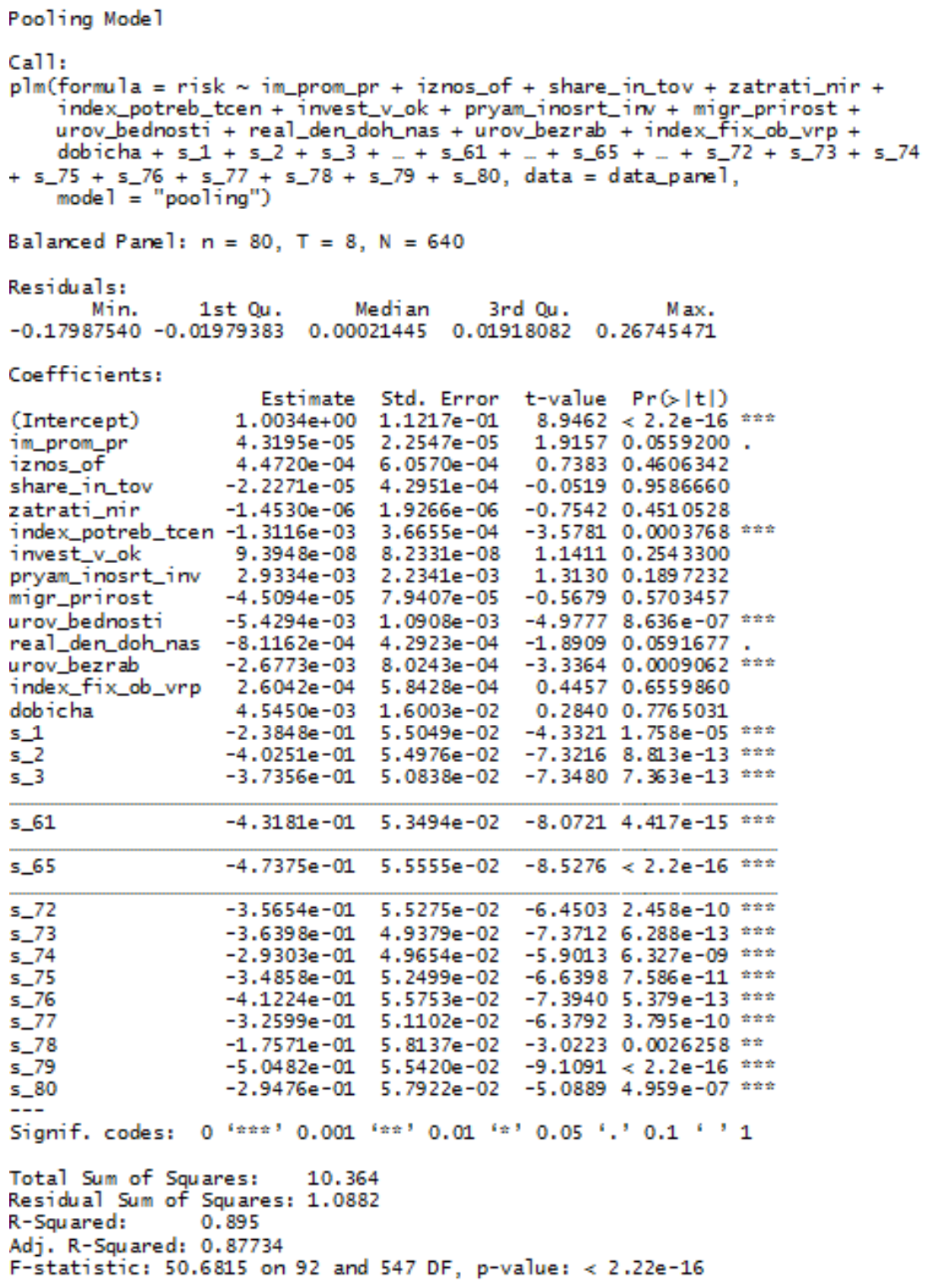

Рис. 9. Модель регрессии с фиксированными эффектами зависимости риска потери потенциала взаимных торговых потоков от выделенных факторов с учётом индивидуальных особенностей субъектов.

Источник: расчёты авторов 
Можно сказать, что безусловно индивидуальные эффекты субъектов оказывают значимое влияние на изменение риска потери потенциала взаимных торговых потоков, в особенности это является актуальным для ДФО ввиду неразвитости инфраструктуры, отдалённости от центральной части России, высокими миграционными оттоками постоянного населения в сочетании с его естественной убылью, недостаточным уровнем жизни населения, а также низкой покупательной способностью жителей региона.

На следующем шаге построим регрессионную модель с фиксированными эффектами, включив в неё два набора дамми-переменных, характеризующих как временные эффекты, так и индивидуальные (рис. 10).

Согласно построенной модели, видно, что значимое влияние на изменение риска потери потенциала взаимных торговых потоков с учётом временных и индивидуальных эффектов оказывают индекс промышленного производства, индекс физического объема ВРП и индекс потребительских цен. Что касается временных фиктивных переменных, то в полученной эконометрической модели в числе незначимых коэффициентов оказались оценки за 2011 и 2017 гг. Также отмечено, что в 2014 г. по отношению к базовому периоду (2010 г.) наблюдается наибольший риск потери потенциала взаимных торговых потоков по причинам, описанным выше.

Таким образом, согласно проведенным оценкам, наиболее предпочтительной при анализе изменчивости риска потери потенциала взаимных торговых потоков в субъектах Российской Федерации является модель с фиксированныли эффектами.

Кроме этого, были построены детализированные модели с фиксированными эффектами с учётом влияния индивидуальных особенностей субъектов России, временных эффектов, а также модель, учитывающая их одновременное влияние.

В каждой из построенных моделей из рассматриваемого апостериорного набора показателей значимое влияние на изменение зависимой переменной оказывал индекс промышленного производства. Данный факт логично объясняется ключевой ролью добывающих производств в экспортных потоках России и её регионов.

Что касается Дальневосточного федерального округа, то можно отметить, что индивидуальные особенности субъектов ДФО (а именно их социально-экономическое развитие) оказывают влияние на снижение риска потери потенциала взаимных торговых потоков. Также на снижение риска в субъектах Дальнего Востока России значимое влияние оказывают темпы прироста индекса промышленного производства, индекс потребительских цен, а также индекс физического объёма валового регионального продукта. Так как ДФО включает в себя преимущественно субъекты с сырьевой направленностью экономики, основным видом экономической деятельности которых является добыча полезных ископаемых, то в регионе, безусловно, необходимо поддерживать потенциал экспортно-сырьевой модели экономического развития. 
Кузнецова Н. В., Кочева Е. В., Матев Н. А. Риск потери потенциала взаимных торговых потоков субъектов Российской Федерации: эконометрическое моделирование

Balanced Pane 1: $n=80, T=8, N=640$

Residuals:

Min. 1st Qu. Median 3rd Qu.

$\begin{array}{lll}0.001705 & 0.017075 & 0.231667\end{array}$

$-0.191323-0.012993$

0.001705

0.017075

Max.

Coefficients:

\begin{tabular}{|c|c|c|c|c|c|}
\hline $\begin{array}{l}\text { (Intercept) } \\
\text { im prom pr }\end{array}$ & $\begin{array}{r}\text { Estimate } \\
7.7434 e-01 \\
8.3714 e-05\end{array}$ & $\begin{array}{l}\text { Std. Error } \\
1.4741 \mathrm{e}-01 \\
2.1967 \mathrm{e}-05\end{array}$ & $\begin{array}{r}\text { t-value } \\
5.2529 \\
3.8109\end{array}$ & $\begin{array}{l}\operatorname{Pr}(>|t|) \\
2.158 e-07 \\
0.0001544\end{array}$ & $\begin{array}{l}* \approx * \\
t \neq *\end{array}$ \\
\hline $\begin{array}{l}\text { znos_of } \\
\text { hare_in_tov } \\
\text { atrati_nir } \\
\text { ndex_potreb_tcer } \\
\text { nvest_v_ok }\end{array}$ & $\begin{array}{r}3.8990 e-04 \\
4.4766 e-04 \\
8.7316 e-08 \\
2.2077 e-03 \\
-5.3318 e-08\end{array}$ & $\begin{array}{l}5.8975 e-04 \\
3.8051 e-04 \\
1.7453 e-06 \\
9.5991 e-04 \\
7.2209 e-08\end{array}$ & $\begin{array}{r}0.6611 \\
1.1765 \\
0.0500 \\
2.2999 \\
-0.7384\end{array}$ & $\begin{array}{l}0.5088188 \\
0.2399237 \\
0.9601176 \\
0.0218317 \\
0.4606034\end{array}$ & \# \\
\hline $\begin{array}{l}\text { ryam_inosrt_inv } \\
\text { igr_prirost } \\
\text { rov_bednosti } \\
\text { eal_den_doh_nas } \\
\text { rov_bezrab }\end{array}$ & $\begin{array}{r}2.0512 e-03 \\
-3.7415 e-06 \\
3.7506 e-04 \\
-7.4149 e-04 \\
-2.9174 e-04\end{array}$ & $\begin{array}{l}1.9624 e-03 \\
7.1345 e-05 \\
1.0349 e-03 \\
5.3985 e-04 \\
7.9317 e-04\end{array}$ & $\begin{array}{r}1.0453 \\
-0.0524 \\
0.3624 \\
-1.3735 \\
-0.3678\end{array}$ & $\begin{array}{l}0.2963715 \\
0.9581960 \\
0.7171829 \\
0.1701594 \\
0.7131538\end{array}$ & \\
\hline $\begin{array}{l}\text { ndex_fix_ob_vrp } \\
\text { lobicha } \\
-1 \\
-2 \\
-3\end{array}$ & $\begin{array}{r}1.0394 e-03 \\
9.9741 e-03 \\
-2.1009 e-01 \\
-3.2461 e-01 \\
-2.9540 e-01\end{array}$ & $\begin{array}{l}5.5453 e-04 \\
1.3982 e-02 \\
4.9375 e-02 \\
5.0091 e-02 \\
4.6111 e-02\end{array}$ & $\begin{array}{r}1.8744 \\
0.7134 \\
-4.2550 \\
-6.4805 \\
-6.4063\end{array}$ & $\begin{array}{l}0.0614126 \\
0.4759224 \\
2.464 e-05 \\
2.063 e-10 \\
3.249 e-10\end{array}$ & $\begin{array}{l}- \\
t \leq t \leq t \\
t t t \\
t t t\end{array}$ \\
\hline-61 & $-3.1686 e-01$ & $5.0221 e-02$ & -6.3093 & $5.844 \mathrm{e}$ & $t=t$ \\
\hline-65 & $-3.5591 \mathrm{e}-01$ & $5.1854 e-02$ & -6.8637 & $1.847 e-11$ & 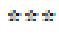 \\
\hline-72 & $-2.7195 e-01$ & $4.9966 e-02$ & -5.4427 & $7.974 e-08$ & 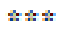 \\
\hline-73 & $-2.6598 e-01$ & $4.5382 e-02$ & -5.8610 & $8.013 e-09$ & 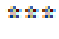 \\
\hline $\begin{array}{l}74 \\
-75 \\
-76\end{array}$ & $\begin{array}{l}-1.9908 e-01 \\
-2.7343 e-01 \\
-3.2564 e-01\end{array}$ & $\begin{array}{l}4.6474 e-02 \\
4.8272 e-02 \\
5.1684 e-02\end{array}$ & $\begin{array}{l}-4.2836 \\
-5.6644 \\
-6.3007\end{array}$ & $\begin{array}{l}2.177 e-05 \\
2.401 e-08 \\
6.155 e-10\end{array}$ & 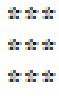 \\
\hline $\begin{array}{l}s \_77 \\
s-78 \\
s-79 \\
s \_80 \\
y \_11\end{array}$ & $\begin{array}{l}-2.8870 \mathrm{e}-01 \\
-1.7111 \mathrm{e}-01 \\
-3.6731 \mathrm{e}-01 \\
-2.9195 \mathrm{e}-01 \\
1.2340 \mathrm{e}-02\end{array}$ & $\begin{array}{l}4.5593 e-02 \\
5.1212 e-02 \\
5.2201 e-02 \\
5.1162 e-02 \\
1.1682 e-02\end{array}$ & $\begin{array}{r}-6.3321 \\
-3.3412 \\
-7.0364 \\
-5.7064 \\
1.0563\end{array}$ & $\begin{array}{l}5.094 \mathrm{e}-10 \\
0.0008920 \\
6.006 \mathrm{e}-12 \\
1.904 \mathrm{e}-08 \\
0.2912964\end{array}$ & 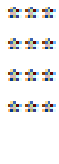 \\
\hline $\begin{array}{l}-12 \\
-13 \\
-14 \\
-15 \\
-16\end{array}$ & $\begin{array}{l}-3.8341 e-02 \\
-5.3488 e-02 \\
-9.0107 e-02 \\
-4.7693 e-02 \\
-2.5131 e-02\end{array}$ & $\begin{array}{l}9.6730 e-03 \\
1.0223 e-02 \\
8.7468 e-03 \\
1.0237 e-02 \\
1.5064 e-02\end{array}$ & $\begin{array}{r}-3.9637 \\
-5.2323 \\
-10.3017 \\
-4.6591 \\
-1.6683\end{array}$ & $\begin{array}{l}8.372 \mathrm{e}-05 \\
2.400 \mathrm{e}-07 \\
<2.2 \mathrm{e}-16 \\
4.004 \mathrm{e}-06 \\
0.0958426\end{array}$ & 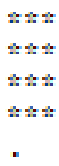 \\
\hline$y \_17$ & $5.2373 e-04$ & $1.6812 e-02$ & 0.0312 & 0.9751594 & \\
\hline Signif. codes: & 'tzt: $\quad 0.001$ & "tzt, 0.01 it & 0.05 & 0.1 & 1 \\
\hline $\begin{array}{l}\text { Total Sum of Squa } \\
\text { Residual Sum of } \\
\text { R-Squared: } \\
\text { Adj. R-Squared: } \\
\text { F-statistic: } 64\end{array}$ & $\begin{array}{l}\text { es: } \quad 10.36 \\
\text { uares: } 0.812 \\
92158 \\
9072 \\
0973 \text { on } 99\end{array}$ & & & & \\
\hline
\end{tabular}

Рис. 10. Модель регрессии с фиксированными эффектами зависимости риска потери потенциала взаимных торговых потоков от выделенных факторов с учётом индивидуальных особенностей субъектов и временных эффектов.

Источник: расчёты авторов 
Пока данный потенциал имеется, регион будет интересен странам АТР. Регион экспортирует в больших объёмах ресурсы, а взамен приобретает продукцию с высокой добавленной стоимостью. Однако ресурсы истощаются, а с расширением технологических возможностей освоения новых видов энергии роль ресурсов в будущем может стремительно снижаться. Именно поэтому субъекты уникального региона крайне нуждаются в реализации государственных программ инновационного развития экономики.

\section{Список литературы}

1. Rodriguez, F. Trade policy and economic growth: a sceptics guide to the cross-national evidence / F. Rodriguez, D. Rodrik. - Cambridge : MIT Press, 2000. URL: https://www.journals.uchicago.edu/doi/pdf/10.1086/654419 (дата обращения: 10.10.2020).

2. Hecksher, E. F. The effect of foreign trade on the distribution of income. Economise Tidskrift. 1919. P. 497-512. Reprinted as Chapter 13 in A.E.A. (1949). Readings in the Theory of international trade, 272-300 (Philadelphia: Blakiston) with a translation in H. Flam and M. J. Flanders (eds.) // Heckscher-Ohlin trade theory. - Cambridge : MIT Press, 1991. - P. 43-69.

3. Ohlin, B. Interregional and international trade / B. Ohlin. - Rev. ed. - Cambridge : Mass., Harvard University Press, 1967. - XVII, 324 p. - (Harvard Economic Studies ; vol. XXXIX).

4. Sapsford foreign direct investment and growth in EP and IS countries / V. N. Balasubramanyam [et al.] // Economic Journal. - 1996. - № 106. - P. 92-105.

5. Wacziarg, R. Measuring the dynamic gains from trade // World Bank Economic Review. - 2001. - Vol. 15, no. 3. - P. 393-429.

6. Caudros, A. Openness and growth: re-examining foreign direct investment, trade and output linkages in Latin America / A. Caudros, V. Orts, M. T. Alguacil // Journal of Development Studies. - 2004. - № 40. - P. 167-192.

7. Baliamoune-Lutz, M. Does FDI contribute to economic growth? // Business Economics. - 2004. - № 39 (2). - P. 49-56.

8. Foreign direct investment and growth in Mediterranean countries / M. Hisarciklilar [et al.] // Conference concluding a Jean Mon-net Project on «Sustainable development and adjustment in the MEDA countries following the EU enlargement», Spetses Island, Greece, 3-5 July, 2006. - URL: http://emo.pspa.uoa.gr/data/pap ers/2_paper.pdf (дата обращения: 10.10.2020).

9. Darrat, A. F. FDI and economic growth in CEE and MENA countries: a tale of two regions / A. F. Darrat, S. Kherfi, M. Soliman. - DOI 10.19030/iber.v4i12.3649 // International Business \& Economics Research Journal (IBER). - 2011. - Vol. 4 (12). 12th Economic Research Forum's Annual Conference, Cairo, Egypt, 2005. - URL: 
Кузнецова Н. В., Кочева Е. В., Матев Н. А. Риск потери потенциала взаимных торговых потоков субъектов Российской Федерации: эконометрическое моделирование

file:///C:/Users/youhi/Downloads/FDI_and_economic_growth_in_CEE_and_MENA_cou ntries_.pdf (дата обращения: 10.10.2020).

10. Alaya, M. Investissement direct étranger et croissance économique : une estimation a partir d'un modèle structurel pour les pays de la rive sud de la méditerranée. Développement Institutions et Analyse de Long terme (DIAL). - [Brighton], 2004. - 35 p.

11. Frankel, J. Does trade cause growth? / J. A. Frankel, D. Romer // American Economic Review. - 1999. - Vol. 89, no. 3. - P. 379-399.

12. Кузнецова, Н. В. Методика оценки социально-экономических рисков для ответа России на большие вызовы / Н. В. Кузнецова, Е. В. Кочева, Н. А. Матев // Азиатско-Тихоокеанский регион: экономика, политика и право. - 2019. № 4. - C. 29-52.

13. Региональные вызовы российского Дальнего Востока : учебное пособие / Н. В. Кузнецова, Е. В. Кочева, Н. А. Матев, А. А. Кравченко ; ДВФУ, ШЭМ. - Владивосток : Изд-во Дальневост. федерал. ун-та, 2019. - 350 с.

\section{References}

1. Rodriguez F., Rodrik D. Trade policy and economic growth: a sceptics guide to the cross-national evidence. Cambridge: MIT Press, 2000. Available at: https://www.journals.uchicago.edu/doi/pdf/10.1086/654419 (accessed 10 October 2020).

2. Hecksher E.F. The effect of foreign trade on the distribution of income. Economise Tidskrift. 1919. P. 497-512. Reprinted as Chapter 13 in A.E.A. (1949). Readings in the Theory of international trade, 272-300 (Philadelphia: Blakiston) with a translation in H. Flam and M. J. Flanders (eds.). Heckscher-Ohlin trade theory. Cambridge: MIT Press, 1991, pp. 43-69.

3. Ohlin B. Interregional and international trade. Rev. ed. Cambridge: Mass., Harvard University Press, 1967. XVII, 324 p. (Harvard Economic Studies ; vol. XXXIX).

4. Balasubramanyam V.N. et al. Sapsford Foreign direct investment and growth in EP and IS countries. Economic Journal, 1996, no. 106, pp. 92-105.

5. Wacziarg R. Measuring the dynamic gains from trade. World Bank Economic Review, 2001, vol. 15, no. 3, pp. 393-429.

6. Caudros A., Orts V., Alguacil M.T. Openness and growth: re-examining foreign direct investment, trade and output linkages in Latin America. Journal of Development Studies, 2004, no. 40, pp. 167-192.

7. Baliamoune-Lutz M. Does FDI contribute to economic growth? Business Economics, 2004, no. 39 (2), pp. 49-56.

8. Hisarciklilar M. et al. Foreign direct investment and growth in Mediterranean countries. Conference concluding a Jean Mon-net Project on «Sustainable development and adjustment in the MEDA countries following the EU enlargement», Spetses Island, 
Greece, 3-5 July, 2006. Available at: http://emo.pspa.uoa.gr/data/papers/2_paper.pdf (accessed 10 October 2020).

9. Darrat A.F., Kherfi S., Soliman M. FDI and economic growth in CEE and MENA countries: a tale of two regions. International Business \& Economics Research Journal (IBER), 2011, vol. 4 (12). DOI: 10.19030/iber.v4i12.3649. Available at: file:///C:/Users/youhi/Downloads/FDI_and_economic_growth_in_CEE_and_MENA_cou ntries_pdf (accessed 10 October 2020).

10. Alaya, M. Foreign direct investment and economic growth: an estimate based on a structural model for the countries of the southern shore of the Mediterranean. Institutional Development and Long-term Analysis (DIAL). 2004. 35 p.

11. Frankel J., Romer D. Does trade cause growth? American Economic Review, 1999, vol. 89, no. 3, pp. 379-399.

12. Kuznetsova N. V., Kocheva E. V., Matev N. A. Metodika otsenki sotsial'noekonomicheskikh riskov dlya otveta Rossii na bol'shie vyzovy [Methods of assessing socio-economic risks for the response of Russia to grand challenges]. AziatskoTikhookeanskii region: Ekonomika. Politika. Pravo - Pacific RIM: Economics. Politics. Law, 2019, no. 4, pp. 29-52.

13. Kuznetsova N. V., Kocheva E. V., Matev N. A., Kravchenko A. A. Regional'nye vyzovy rossiiskogo Dal'nego Vostoka: uchebnoe posobie [Regional challenges of the Russian Far East: textbook. FEFU, School of Economics and Management]. Vladivostok: Far Eastern Federal University Publishing House, 2019. 350 p. 